\title{
Bowel habits and gender correlate with colon length measured by CT colonography
}

\author{
Kenichi Utano ${ }^{1} \cdot$ Koichi Nagata $^{2} \cdot$ Tetsuro Honda $^{3} \cdot$ Takashi Kato $^{4} \cdot$ Alan Kawarai Lefor ${ }^{5} \cdot$ Kazutomo Togashi $^{1}$ (]
}

Received: 12 June 2021 / Accepted: 23 September 2021 / Published online: 11 October 2021

(c) The Author(s) 2021

\begin{abstract}
Purpose CT colonography enables three-dimensional measurement of colon length. However, previous studies using CT colonography have not examined the association with gender, age, physique, a history of laparotomy and bowel habits, all possible contributory factors to colon length. The aim of this study is to investigate factors associated with colon length. Materials and methods We conducted a post hoc analysis based on data obtained from a previous multi-center trial including 321 patients with positive fecal immunochemical tests who underwent CT colonography. Colon length was measured using a computer-generated center line and was divided at the iliac crest level into the distal and proximal colons. Bowel habits were classified into three groups: A-daily; B-once every 2 or 3 days; and C-less than once in 3 days. Statistical comparison was made using one-way ANOVA with Bonferroni's correction.

Results A total of 295 patients were analyzed. The entire colon length $(\mathrm{cm}$, mean \pm standard deviation) of individual patients was $150.3 \pm 18.5 \mathrm{~cm}$ and ranged from 109.7 to $195.9 \mathrm{~cm}$. The female colon was significantly longer than the male colon $(154.3 \pm 18.1 \mathrm{~cm}$ vs. $147.1 \pm 18.3 \mathrm{~cm} ; p=0.022)$. Colon length showed trends associated with age $(p=0.18)$ and a history of laparotomy $(p=0.14)$. According to bowel habits, the entire colon measured $147.4 \pm 17.9$ in group A, $154.7 \pm 18.5$ in group B and 158.6 \pm 18.3 in group C, and significant differences were observed for "A vs. C" ( $p=0.002)$ and "A vs. B" $(p=0.014)$. In subgroup analysis by colon segment, the proximal colon trended similarly to the entire colon while there were no trends for the distal colon.

Conclusions This study has clearly demonstrated that bowel habits and gender both correlate with the length of the entire colon measured by CT colonography, and in particular, the proximal colon.

Secondary abstract Using CT colonography, we measured the colon length in 295 patients. The entire colon length was $150.3 \pm 18.5 \mathrm{~cm}$ on average. Females and constipated (less frequent defecation) patients have a significantly longer colon, and in particular, the proximal colon. Colon length showed trends associated with age and a history of laparotomy.
\end{abstract}

Keywords Computed tomographic colonography $\cdot$ Gender $\cdot$ Constipation $\cdot$ Defecation

Kazutomo Togashi

togashik@fmu.ac.jp

1 Department of Coloproctology, Aizu Medical Center, Fukushima Medical University, 21-2 Maeda, Tanisawa, Kawahigashi, Aizuwakamatsu, Fukushima 969-3492, Japan

2 Department of Gastroenterology, Fukushima Medical University, Fukushima, Japan

3 Department of Gastroenterology, Nagasaki Harbor Medical Center, Nagasaki, Japan

4 Department of Internal Medicine, Hokkaido Gastroenterology Hospital, Sapporo, Japan

5 Department of Surgery, Jichi Medical University, Shimotsuke, Japan

\section{Introduction}

Constipation is one of the most common chronic gastrointestinal conditions, which reportedly affects $14-17 \%$ of the population $[1,2]$, more frequently in females and the elderly [3], and adversely affects both physical and psychological quality of life [4]. In general, endoscopists have empirical knowledge that difficult colonoscopy is frequently encountered in elderly, thin or female patients, or who have constipated bowel habits, a history of laparotomy, or diverticular disease $[5,6]$, and that difficult colonoscopy is mostly attributed to a long and redundant colon [7].

Since the nineteenth century, there have been many studies of the anatomy of the large intestine at autopsy [8-10]. 
These cadaver studies attempted to elucidate the association of colon length with age, gender and physique, and showed inconsistent results probably due to varying degrees of postmortem rigidity after death. In 1995, Saunders et al. reported variations in colon length in 118 patients undergoing laparotomy, measuring according to a set protocol with the bowel pulled medially, mimicking the possible displacements that may occur during colonoscopy [11]. However, this measurement method possesses intrinsic limitations, e.g., inability to measure a colon with adhesions or a distal rectum, resulting in a shorter colon than using other methods. Barium enema examination allows two-dimensional estimation of the colon length mainly using an opisometer (mapping wheel). Despite the rough estimates, this measurement method found a correlation of colon length with colon transit time [12], difficult colonoscopy [13], incidence of sigmoid volvulus [14], age and gender [15]. Colonic transit time, that is, an objective test to diagnose slow transit constipation and other gastrointestinal symptoms correlated with colon redundancy rated in a non-quantitative manner [12]. In another study to investigate colon anatomy and motility in young women with severe idiopathic constipation [16], the colon was longer in constipated women $(n=37)$ than in controls $(n=20)$, but the difference was not significant probably due to insufficient statistical power of the study.

With the recent emergence of computed tomography (CT) colonography, three-dimensional measurement of colon length is feasible. This means that physicians can easily obtain information on the actual colon length of live patients. So far, several researchers have measured colon length using CT colonography to identify the cause of incomplete colonoscopy [17], colon length difference compared with colonoscopy $[18,19]$, positional alteration of the colon length [20] and the correlation of colon anatomy with age, gender and body mass index [21]. Ohgo et al. reported that the entire colon in patients with functional constipation was significantly longer than patients with diarrheatype irritable bowel syndrome (IBS) or control patients in a small-scale case-control study [22]. However, previous studies using CT colonography have not examined the association with gender, age, physique, a history of laparotomy and bowel habits, all possible contributory factors to colon length. The aim of this study was to comprehensively investigate factors which correlate with colon length in a large observational cohort, particularly focused on bowel habits.

\section{Materials and methods}

\section{Study design}

This study adopted a post hoc design, using data obtained from a previous multi-center trial (UMIN Clinical Trials
Registry number UMIN000006665) including 321 patients with positive fecal immunochemical tests (FIT) who underwent CT colonography [23], which was performed in seven hospitals across Japan. Ethical review approval was obtained from all seven hospitals and all patients gave their written informed consent, including for this post hoc analysis.

\section{Patients}

Patients were eligible for inclusion into the study if they were 40 years of age or older and were seen at participating institutions to undergo colonoscopy for colorectal cancer examination because of a recent positive FIT result. Patients were excluded for any of the following reasons: serious comorbidity; a history of colon screening tests in the preceding 3 years; known colorectal neoplasms; a history of inflammatory bowel disease, Lynch syndrome, familial polyposis, or colorectal surgery; pregnancy, hyperthyroidism, or iodinated contrast medium allergy. Eligible consecutive patients were prospectively enrolled in the study between December 2011 and September 2012.

Prior to enrollment, information on bowel habits was obtained through an interview by nursing staff. Bowel habits were classified into three categories according to the frequency of bowel movements: daily group, every day; intermediate group, once every 2 or 3 days; and constipated group, less than once in 3 days. Patients' information on age, gender, height, weight, body mass index (BMI) and history of laparotomy were also obtained. Patients status-post gastrointestinal or uterus resection were classified as major, and those without such resections (e.g., appendectomy) were classified as minor laparotomies.

\section{CT colonography}

Preparation started 1 day before CT colonography, and, patients received $380 \mathrm{~mL}$ polyethylene glycol solution (Niflec; Ajinomoto Pharmaceuticals, Japan), $20 \mathrm{~mL}$ iodinated oral contrast agent for fecal tagging, and two doses of $20 \mathrm{mg}$ mosapride (Gasmotin; Sumitomo Dainippon Pharma, Japan) the day before CT colonography after breakfast and dinner. On the examination day, patients were placed in the left decubitus position for thin flexible rectal-tube insertion with a balloon. The colon was insufflated using an automated carbon dioxide insufflator prototype (HP-2, Horii Pharmaceutical Ind, Japan) and insufflation pressure was set to $20 \mathrm{mmHg}$. The total volume of carbon dioxide insufflated was recorded. All CT colonography examinations at all institutions were performed using 16- or 64-row multidetector CT scanner, with supine and prone positioning. The scanning protocol was as follows: $120 \mathrm{kVp}$, automatic tube current modulation system or a tube current of $50 \mathrm{~mA}$, 
and a section thickness $\leq 1.0-\mathrm{mm}$. Spasmolytic agents and intravenous contrast media were not used during CT colonography [24].

\section{Image analysis}

All CT colonography images were analyzed by an experienced radiologist who had over 10 years of abdominal diagnostic radiology experience. As part of image processing, the workstation automatically creates a centerline for 3D fly-through image review. We recorded the distance of the centerline from the anus to the cecal tip. All interpretations were performed using a commercially available workstation (AZE Virtual Place, Canon Medical System, Japan). Colon length was measured in two positions: the supine position and the prone position, and the average of the two measurements was defined as the colon length of each subject. The entire colon length was the distance from the anus to the cecal tip, and the colon was divided into two parts (proximal colon: cecum to descending colon, distal colon: sigmoid colon and rectum). The boundary between the descending colon and sigmoid colon was at the height of the iliac crest.

\section{Outcome measurements}

The primary outcome is the mean entire colon length of the two measurements in the supine and prone positions, by age group (40-59, 60-79, 80 years or older), gender, body mass index (underweight: -18.5 , normal weight: $18.5-25$, overweight: 25-), history of laparotomy (major, minor, none) and bowel habits (daily, intermediate, constipated). Secondary outcomes are proximal colon length and distal colon length, using the same factors mentioned above.

\section{Statistical analysis}

The skewness and kurtosis test was used to test for a normal distribution of colon length. If normal distribution is confirmed, colon length is expressed as mean \pm standard deviation (SD) and compared using one-way ANOVA with Bonferroni's correction for multiple comparisons. Otherwise, colon length is expressed as median [interquartile range] and compared using nonparametric tests. A multivariate linear regression model was applied to analyze factors correlating with colon length. Categorical variables were presented as percentages and numbers and were compared by the $\chi^{2}$ test or Fisher exact test if appropriate. The threshold for significance was $p<0.05$. All statistical analyses were performed with Intercooled Stata 16.0 ${ }^{\mathrm{TM}}$ for Windows (Stata Corp. USA).

\section{Results}

\section{Study patients}

After excluding patients with poor bowel preparation, insufficient insufflation of the colorectum during CT colonography acquisition, or with a history of bowel resection and advanced cancer (stage T2: proper muscle layer or beyond), 295 patients (mean age $58.0 \pm 11.0$ years, range $40-83$ years; female 141) were enrolled (shown in Fig. 1). The average total volume of carbon dioxide insufflated was $2612 \mathrm{~mL}$ (range: 1200-6900 mL). The characteristics of the patients are summarized in Table 1 . The characteristics of patients by gender is shown in Supplementary Table 1. Height, weight and BMI were significantly greater in male patients, compared with females. Females had a significantly higher proportion undergoing major surgery and having constipated bowel habits. Bowel habits classified according to defecation frequency revealed $64 \%$ in the daily group, $23 \%$ in the intermediate group and $13 \%$ in the constipated group. Characteristics of patients by bowel habits are shown in supplementary Table 2. Age, BMI and a history of laparotomy have no significant differences. In contrast, the proportion of patients with "daily" bowel habits was significantly lower in females compared with males ("daily" vs. "intermediate": $p<0.001$; "daily" vs. "constipated": $p<0.001)$. Patients with "daily" bowel habits had a trend toward higher weight (vs. "intermediate": $p=0.077$; vs. "constipated": $p=0.01$ ) and significantly greater height compared to other bowel habit patterns (vs. "intermediate": $p=0.036$; vs. "constipated": $p=0.001$ ).

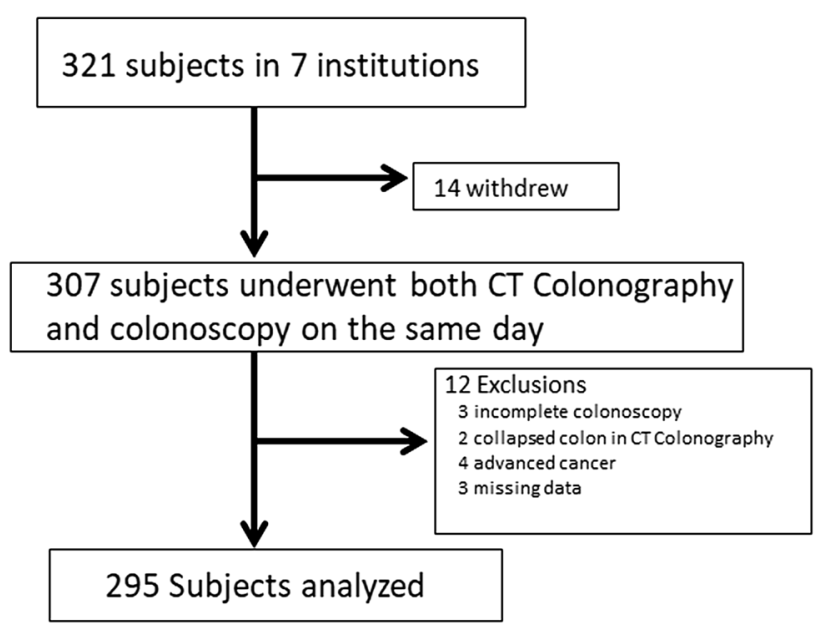

Fig. 1 Study flow diagram. CTC computed tomography colonography 
Table 1 Study patients' characteristics

\begin{tabular}{ll}
\hline Gender & \\
Male, $n(\%)$ & $154(52 \%)$ \\
Female, $n(\%)$ & $141(48 \%)$ \\
Age, years & \\
Mean \pm SD & $58.0 \pm 11.0$ \\
$\quad 40-59, n(\%)$ & $163(55 \%)$ \\
$\quad 60-79, n(\%)$ & $121(41 \%)$ \\
80 or older, $n(\%)$ & $11(3 \%)$ \\
Weight, kg & \\
Mean \pm SD & $60.2 \pm 11.1$ \\
Height, cm & \\
Mean \pm SD & $161.4 \pm 8.7$ \\
Body mass index, kg/m ${ }^{2}$ & \\
Mean \pm SD & $23 \pm 3.3$ \\
History of laparotomy & \\
Major, $n(\%)$ & $15(5.1 \%)$ \\
Minor, $n(\%)$ & $72(24 \%)$ \\
None, $n(\%)$ & $208(71 \%)$ \\
Bowel habits & $189(64 \%)$ \\
Daily, $n(\%)$ & $68(23 \%)$ \\
Intermediate, $n(\%)$ & $38(13 \%)$ \\
Constipated, $n(\%)$ & \\
\hline Bowe & \\
\hline
\end{tabular}

Bowel habits were divided into three groups by defecation frequency: daily group, every day; intermediate group, once every 2 or 3 days; and constipated group, less than once in 3 days. History of laparotomy: those who underwent gastro-intestinal or uterus resections were classified as major and others were classified as minor

$S D$ standard deviation

\section{Colon length and associated factors (shown in Table 2)}

The length of the entire colon had a normal distribution (shown in Fig. 2), which was confirmed by a skewness and kurtosis test $(p=0.104)$. The entire colon length $($ mean $\pm \mathrm{SD})$ of individual patients was $150.3 \pm 18.5 \mathrm{~cm}$ and ranged from 109.7 to $195.9 \mathrm{~cm}$. The entire colon of the "80 years or older" age group had a trend toward being longer, but significant differences were not observed in any comparisons (vs. "40-59": $p=0.203$; vs. "60-79": $p=0.335$ ). In contrast, the female colon is significantly longer than the male colon ( $p=0.022$, shown in Fig. 3a). Any group categorized according to BMI had almost the same colon length. The "major" laparotomy group had a trend toward a longer colon, but there were no significant difference among any comparisons. Colon lengths by bowel habits were significantly different $(p=0.003$, shown in Fig. 3b). The entire colon in the constipated group was significantly longer than in the daily group $(p=0.002)$, but not significantly different from the intermediate group $(p=0.877)$. The colon in the intermediate group was significantly longer than in the daily group ( $p=0.014)$. Examples according to bowel habits are shown in Fig. 4a, b, c.

In subgroup analysis by segment of colon (shown in Table 2), the proximal colon trended similarly as the entire colon (shown in Fig. 3c, d), but there were no trends observed for the distal colon (shown in Fig. 3e, f). The female proximal colon is significantly longer than the male proximal colon $(p=0.0003)$. The proximal colon in the constipated group was significantly longer than in the daily group ( $p=0.001)$. The proximal colon of patients undergoing major surgery had a trend toward being longer but only comparison between major and minor previous laparotomies approached a statistically significant difference $(p=0.051)$.

In subgroup analysis by gender (Table 3 ), female colon length by bowel habits was significantly different $(p=0.022)$. The female entire colon in the constipated group was significantly longer than in the daily group $(p=0.026)$, but there was no significant difference between the constipated group and intermediate group $(p=0.93)$ and between the intermediate group and daily group $(p=0.25)$. In contrast, significant differences were not observed in any comparisons in male colon $(p=0.26)$.

\section{Linear regression analysis of factors associated with colon length (shown in Table 4)}

In multivariate linear regression analyses of the length of the entire colon, significant differences were observed for gender $(p=0.002)$ and bowel habits $(p=0.003)$. Age approached statistical significance $(p=0.053)$. In analyses of the length of the proximal colon, similar trends were observed, and age was significantly different $(p=0.017)$. Height was significant in univariate analysis (shown in Supplementary Table 3), but not in multivariate analysis. For distal colon length, only bowel habits were significantly different in univariate analysis (shown in Supplementary Table 3) but not in multivariate analysis.

\section{Discussion}

This study using CT colonography shows that females and constipated (less frequent defecation) patients have a significantly longer colon, and that these trends also are shown in analyses limited to the length of the proximal colon. However, colon length is not associated with physique, regardless of gender. These observations are confirmed by two 
Table 2 Colon length by variables correlating with colon length

\begin{tabular}{|c|c|c|c|c|c|c|c|}
\hline & $N$ & $\begin{array}{l}\text { Entire colon length, } \\
\mathrm{cm}(\text { mean } \pm \mathrm{SD})\end{array}$ & $p$ value* & $\begin{array}{l}\text { Proximal colon length, } \\
\mathrm{cm}(\text { mean } \pm \mathrm{SD})\end{array}$ & $p$ value* & $\begin{array}{l}\text { Distal colon length, } \\
\mathrm{cm}(\text { mean } \pm \mathrm{SD})\end{array}$ & $p$ value* \\
\hline All & 295 & $150.3 \pm 18.5$ & & $90.1 \pm 12.9$ & & $60.4 \pm 11.3$ & \\
\hline \multicolumn{8}{|l|}{ Age (years) } \\
\hline $40-59$ & 163 & $149.6 \pm 17.2$ & 0.18 & $89.2 \pm 12.2$ & 0.10 & $60.5 \pm 10.8$ & 0.74 \\
\hline $60-79$ & 121 & $150.9 \pm 19.5$ & & $90.7 \pm 13.4$ & & $60.1 \pm 11.9$ & \\
\hline 80 or older & 11 & $160.2 \pm 25.2$ & & $97.3 \pm 17.9$ & & $62.9 \pm 13.4$ & \\
\hline \multicolumn{8}{|l|}{ Gender } \\
\hline Male & 154 & $147.1 \pm 18.3$ & 0.022 & $87.5 \pm 12.9$ & 0.0003 & $59.5 \pm 11.5$ & 0.17 \\
\hline Female & 141 & $154.3 \pm 18.1$ & & $92.9 \pm 12.5$ & & $61.4 \pm 11.1$ & \\
\hline \multicolumn{8}{|c|}{ Body mass index } \\
\hline$<18.5$ & 18 & $151.3 \pm 18.7$ & 0.73 & $90.2 \pm 15.8$ & 0.17 & $61.1 \pm 13.1$ & 0.64 \\
\hline$\geq 18.5,<25$ & 201 & $150.0 \pm 18.2$ & & $89.3 \pm 11.8$ & & $60.7 \pm 11.2$ & \\
\hline$\geq 25$ & 74 & $151.9 \pm 19.6$ & & $92.6 \pm 14.9$ & & $59.3 \pm 11.3$ & \\
\hline \multicolumn{8}{|c|}{ History of laparotomy } \\
\hline Major & 15 & $156.7 \pm 17.5$ & 0.14 & $96.7 \pm 14.3$ & 0.051 & $59.9 \pm 10.8$ & 0.56 \\
\hline Minor & 72 & $149.7 \pm 17.5$ & & $87.9 \pm 11.8$ & & $61.7 \pm 9.5$ & \\
\hline None & 208 & $150.4 \pm 18.9$ & & $90.4 \pm 13.1$ & & $60.0 \pm 11.9$ & \\
\hline \multicolumn{8}{|l|}{ Bowel habits } \\
\hline Daily & 189 & $147.7 \pm 17.9$ & 0.003 & $88.2 \pm 12.6$ & 0.001 & $59.2 \pm 11.1$ & 0.058 \\
\hline Intermediate & 68 & $154.7 \pm 18.5$ & & $92.0 \pm 12.8$ & & $62.7 \pm 12.0$ & \\
\hline Constipated & 38 & $158.6 \pm 18.3$ & & $96.3 \pm 12.8$ & & $62.2 \pm 10.5$ & \\
\hline
\end{tabular}

Colon was divided into proximal segment (cecum to descending colon) and distal segment (sigmoid colon and rectum) at the height of the iliac crest. History of laparotomy was classified in major (e.g., gastro-intestinal or uterus resection), minor (e.g., appendectomy) and none. Bowel habits were classified into a daily group (every day), an intermediate group (once every 2 or 3 days) and a constipated group (less than once in 3 days)

$S D$ standard deviation

*Calculated using one-way ANOVA

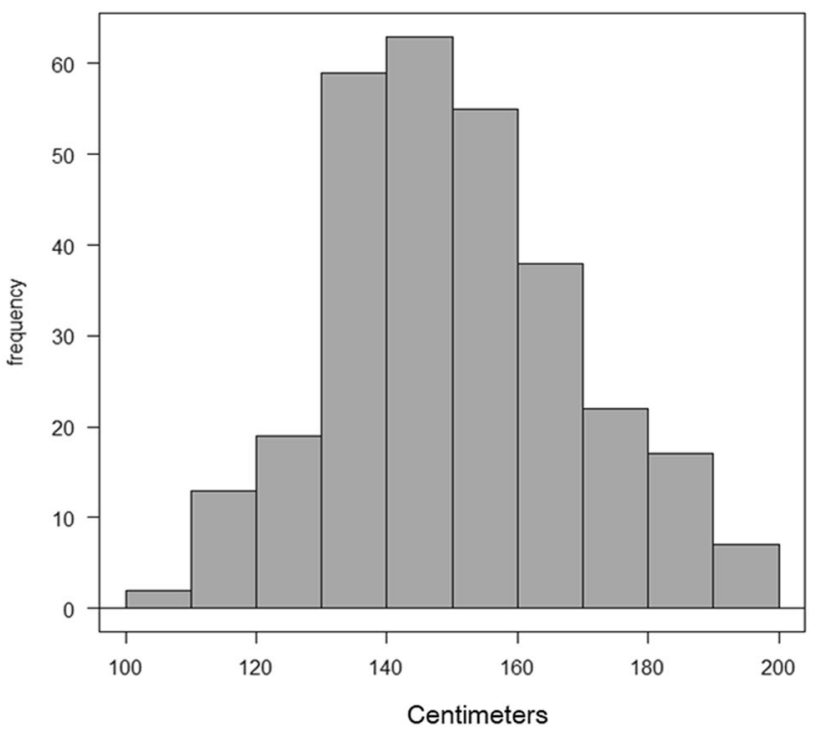

Fig. 2 Histogram of mean entire colon length: the length of the entire colon had a normal distribution, which was confirmed by a skewness and kurtosis test $(p=0.104)$ different statistical methods. To our knowledge, this is the first study to show that bowel habits (defecation frequency) clearly correlate with colon length in a cohort study. A previous case-control study $(n=51)$ utilizing CT colonography reported that patients with functional constipation had a significantly longer colon compared with diarrhea type IBS or control patients [22]. These results suggest that defecation frequency is associated with colon length and are consistent with the results of the present study.

The difference in colon length between "daily" and "constipated" groups was an average of $11 \mathrm{~cm}$ and the difference between the "daily" and "intermediate" groups was an average of $7 \mathrm{~cm}$. It is important to consider how such seemingly small difference could affect defecation frequency. A plausible assumption is that constipated bowel habits could lengthen the colon as a result of fecal impaction. According to a recent murine physiology study, however, elongation of colon by longitudinally stretching the colonic muscle has the effect of inhibiting the colonic migrating motor complexes which are responsible for fecal pellet propulsion in the murine large bowel, finally leading to colonic accommodation and slow transit [25]. However, a partially obstructed 

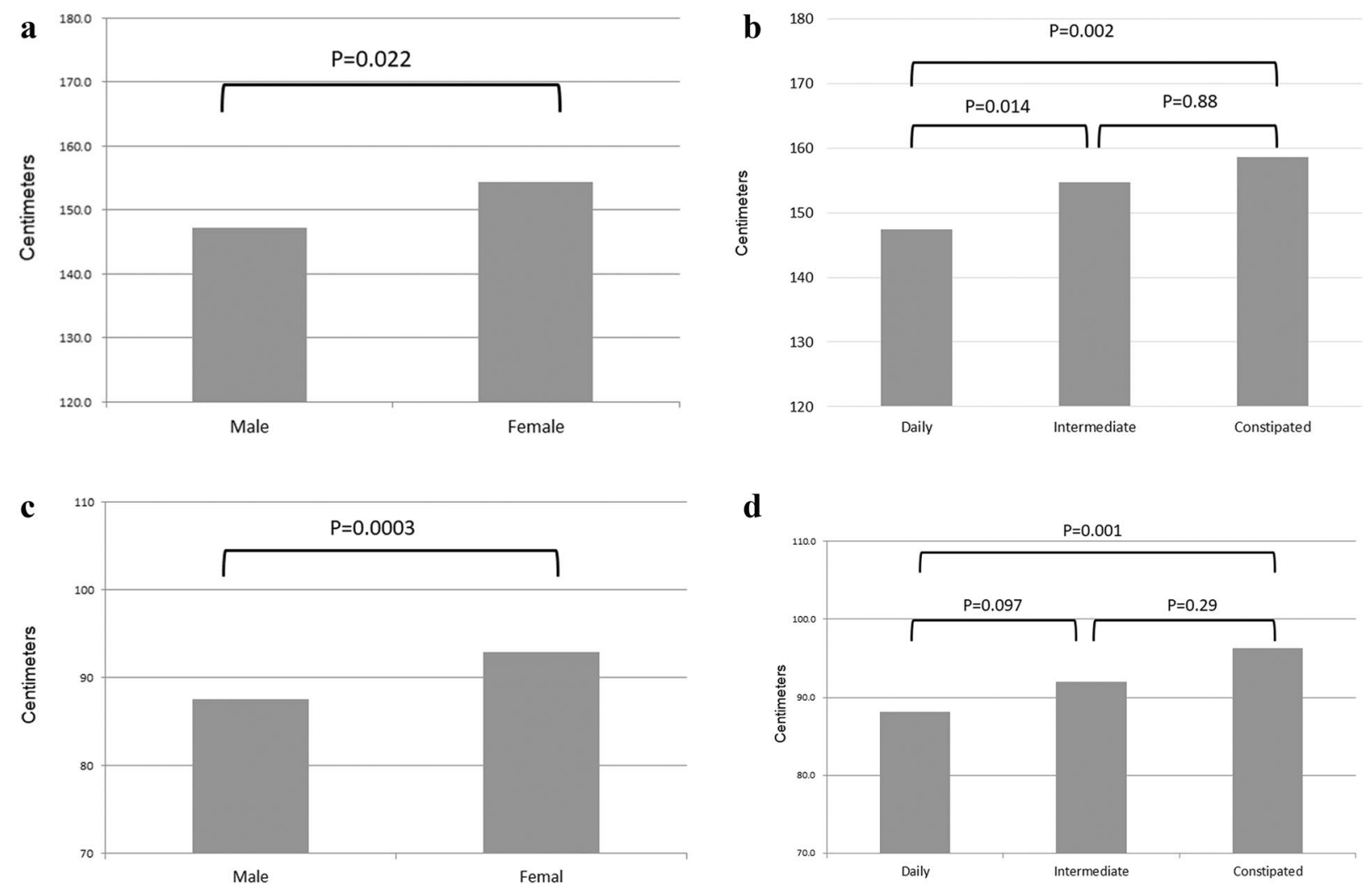

d
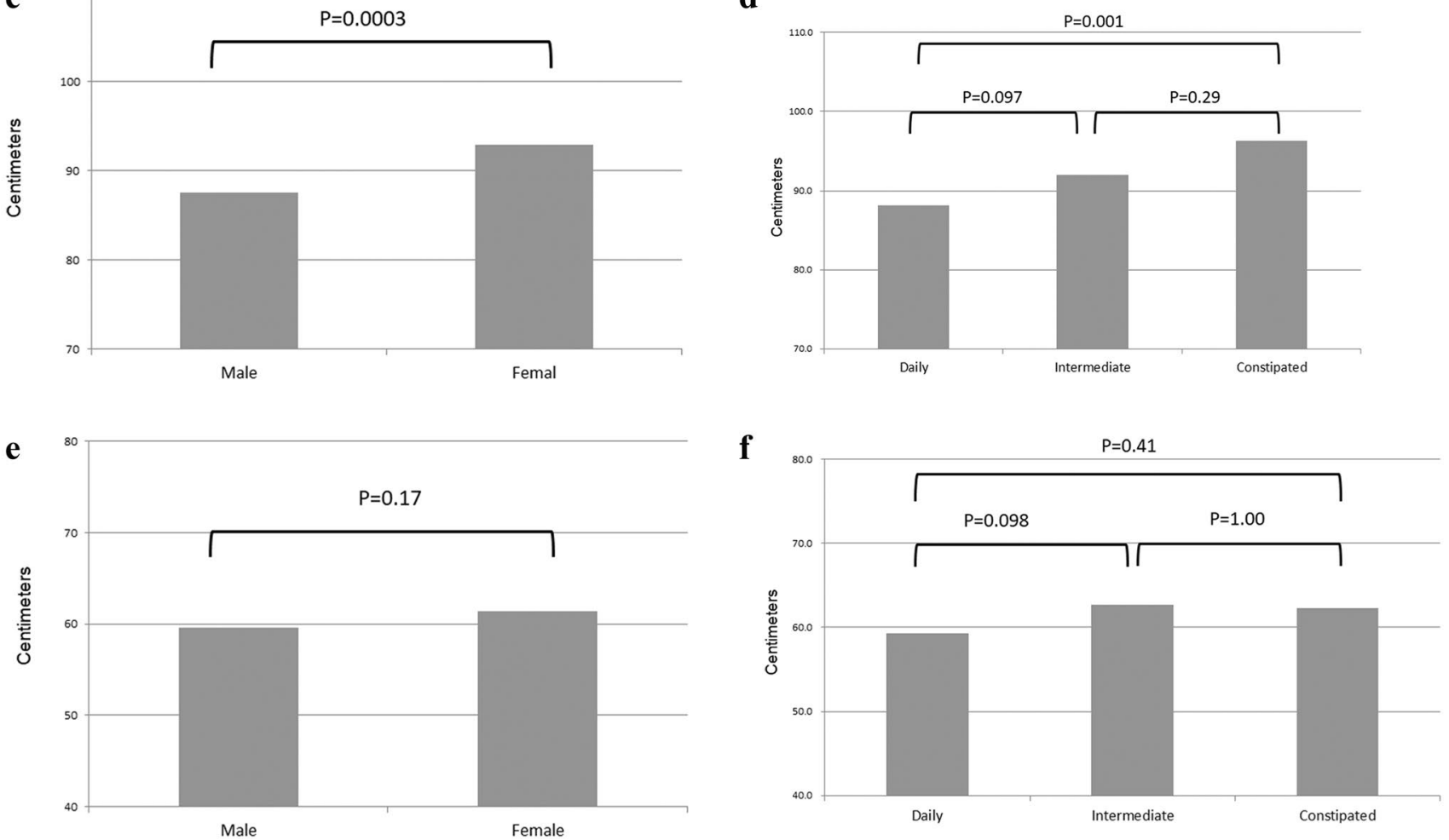

Fig. 3 a Entire colon length by gender. b Entire colon length by bowel habits. c Proximal colon length by gender. d Proximal colon length by bowel habits. e Distal colon length by gender. $\mathbf{f}$ Distal colon length by bowel habits

outlet in a murine colon resulted in an elongated impacted colon with slowed transit [26]. A similar mechanism may be active in the human colon.

In the present study, proximal colon elongation was present while distal colon elongation was not present in patients classified as constipated. In a nuclear scintigraphy study in children, although unable to quantify colon length, transverse colon elongation is more common but sigmoid colon elongation is not present in those with slow-transit constipation [27]. Even in adults, slow transit constipation could influence proximal colon elongation. To diagnose slow transit constipation, objective tests, e.g., daily stool weight $(<35 \mathrm{~g} / \mathrm{d})$, colonic transit time, and anorectal function are required, and diagnostic evaluations should be performed while the patient is not taking laxatives [28]. These objective tests may be onerous for patients to undergo. In addition to the Bristol Stool Form Scale which can estimate colonic transit time [29], proximal colon length measured by CT colonography might be a new surrogate biomarker to identify slow transit constipation.

Notably, the present study has clearly demonstrated that the female colon is longer than the male colon, consistent with two previous relatively large-scale cohort studies; one 

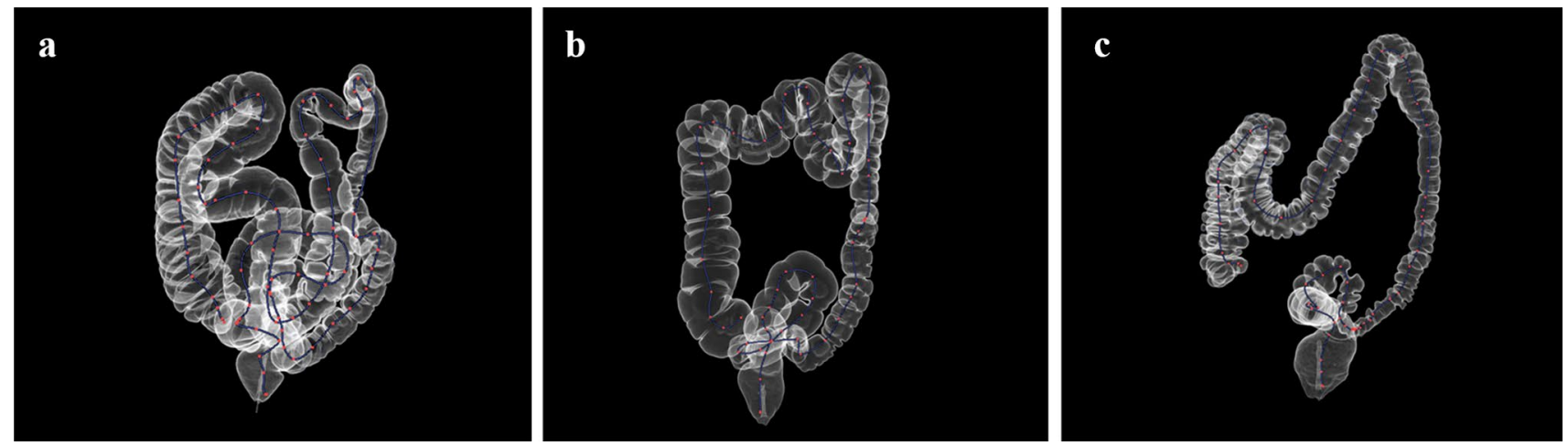

Fig. 4 a Constipated group (defecation: less than once in 3 days). 65 years old, female, body height $151 \mathrm{~cm}$, body weight $48 \mathrm{~kg}$, BMI 21.0. The colon is long and redundant (entire colon $190.9 \mathrm{~cm}$, proximal colon $114.6 \mathrm{~cm}$, distal colon $76.3 \mathrm{~cm}$, in the supine position). b Intermediate group (defecation: once every 2 or 3 days). 53 years old, female, body height $154 \mathrm{~cm}$, body weight $55 \mathrm{~kg}$, BMI 23.2. The colon looks slightly long (entire colon $152.2 \mathrm{~cm}$, proximal colon $82.9 \mathrm{~cm}$, distal colon $69.3 \mathrm{~cm}$, in the supine position). c Daily group (defecation: every day). 61 years old, male, body height $177 \mathrm{~cm}$, body weight $57 \mathrm{~kg}$, BMI 18.1. The colon looks short (entire colon $115.4 \mathrm{~cm}$, proximal colon $67.7 \mathrm{~cm}$, distal colon $47.7 \mathrm{~cm}$, in the supine position)

Table 3 Colon length by gender and bowel habits

\begin{tabular}{|c|c|c|c|c|c|c|c|c|}
\hline & \multicolumn{4}{|l|}{ Male } & \multicolumn{4}{|l|}{ Female } \\
\hline & Daily & Intermediate & Constipated & $p$ value* & Daily & Intermediate & Constipated & $p$ value* \\
\hline Entire colon Length (cm) & $145.8 \pm 18.3$ & $151.9 \pm 17.1$ & $150.9 \pm 17.3$ & 0.26 & $150.2 \pm 16.6$ & $156.3 \pm 18.9$ & $160.6 \pm 17.7$ & $0.022 *$ \\
\hline Proximal colon length $(\mathrm{cm})$ & $86.7 \pm 13.1$ & $90.8 \pm 11.1$ & $90.0 \pm 13.4$ & 0.31 & $90.8 \pm 11.3$ & $92.7 \pm 13.5$ & $98.0 \pm 11.9$ & $0.021 *$ \\
\hline Distal colon length $(\mathrm{cm})$ & $59.1 \pm 11.7$ & $61.2 \pm 11.8$ & $60.9 \pm 7.1$ & 0.68 & $59.5 \pm 10.0$ & $63.5 \pm 12.0$ & $59.5 \pm 10.0$ & 0.130 \\
\hline
\end{tabular}

Proximal colon: cecum to descending colon. Distal colon: sigmoid colon and rectum. Bowel habits were classified in a daily group (every day), an intermediate group (once every 2 or 3 days) and a constipated group (less than once in 3 days)

*Calculated using one-way ANOVA

Table 4 Multivariate linear regression analysis for variables correlating with colon length

\begin{tabular}{|c|c|c|c|c|c|c|c|c|c|}
\hline & \multicolumn{3}{|l|}{ Entire colon } & \multicolumn{3}{|c|}{ Proximal colon } & \multicolumn{3}{|l|}{ Distal colon } \\
\hline & Coefficients & $95 \% \mathrm{CI}$ & $p$ value & Coefficients & $95 \% \mathrm{CI}$ & $p$ value & Coefficients & $95 \% \mathrm{CI}$ & $p$ value \\
\hline Age & 0.22 & 0.00 to 0.45 & 50.053 & 0.19 & 0.03 to 0.35 & 0.017 & 0.031 & -0.11 to 0.17 & 0.66 \\
\hline Gender (M 1, F 2) & 10.8 & 3.9 to 17.6 & 0.002 & 8.8 & 4.0 to 13.5 & $<0.001$ & 2.0 & -2.3 to 6.3 & 0.37 \\
\hline Weight & -0.0041 & -2.0 to 2.0 & 0.997 & -0.57 & -0.36 to 1.80 & 0.42 & 0.57 & -0.71 to 1.84 & 0.38 \\
\hline Height & 0.38 & -1.2 to 1.9 & 0.63 & 0.72 & -0.90 to 0.61 & 0.19 & -0.34 & -1.33 to 0.65 & 0.50 \\
\hline Body Mass Index & 0.40 & -4.9 to 5.7 & 0.88 & 2.0 & -1.7 to 5.6 & 0.29 & -1.6 & -4.9 to 1.8 & 0.35 \\
\hline History of surgery & 0.057 & -3.6 to 3.8 & 0.98 & -0.78 & -3.35 to 1.80 & 0.55 & 0.83 & -1.51 to 3.18 & 0.49 \\
\hline Bowel habits & 4.7 & 1.6 to 7.7 & 0.003 & 2.9 & 0.8 to 5.1 & 0.007 & 1.7 & -0.2 to 3.7 & 0.079 \\
\hline
\end{tabular}

Colon was divided into proximal segment and distal segment at the height of the iliac crest. History of laparotomy was classified in major (2), minor (1) and none (0). Bowel habits were classified into a daily group (1), an intermediate group (2) and a constipated group (3)

$C I$ confidence interval, $M$ male, $F$ female

study applied CT colonography to asymptomatic American patients $(n=505)$ [21] and another used barium enema examination for Japanese patients $(n=920)$ [15]. Sex hormones can affect bowel transit time in women during different stages of their menstrual cycle, although they do not appear to have a major effect on bowel function under normal physiological conditions [30]. During childhood, constipation is common in boys, but is much more common in female adults aged $15-50$ years, that is, during their reproductive years [30]. In addition, women with severe constipation have a high incidence of having undergone gynecological surgery [30]. In the present study, indeed, female 
patients had a higher prevalence of undergoing laparotomy and patients with major laparotomy had a trend toward a longer proximal colon. These observations imply that hormonal and/or gynecological changes are associated with bowel habits and may subsequently alter the colon length.

Measurement methods other than CT colonography have individual drawbacks in nature, as mentioned above in the introduction. At present, CT colonography is the most reliable method to measure actual colon length. However, previous studies using CT colonography showed various values for the length of the entire colon, as summarized in Table 5 $[17-19,21,31]$. It is obvious that patients with incomplete colonoscopy have a long colon [17]. The colon in Americans appears longer than in Japanese patients, but we must consider differences in the measurement software used in the USA and Japan. As part of image processing, both software packages (V3D Colon, Viatronix Inc., USA, and AZE Virtual Place, Canon Medical System, Japan) automatically create a centerline that serves as the focal point for three-dimensional fly-through image review. The length of the centerline from the anus to the cecal tip represents the length of the entire colon, but the centerlines automatically generated depend on the software used. For instance, a submerged lumen with fecal fluid and collapsed segments of colon are not identically considered by the different software, and the software is evolving year by year, causing distinct differences. Nagata et al. analyzed the American colon using an open-access database (the Cancer Image Archive) and reported that the total colorectal length in Americans $(n=650)$ was significantly longer than in Japanese $(n=650)$ but the difference was very small [31], which suggests that racial difference in colon length likely do not exist. Considering that colon length was not associated with height in a previous study [21] as well as in the present study, the difference between American and Japanese colon lengths is negligible.

This study has several acknowledged limitations. First, this is a post hoc analysis using data obtained from a previous multi-center trial, and the sample size was not calculated. Second, all patients were Japanese manifesting positive FIT, and the generalizability of these findings may be limited. Third, we did not apply the Rome IV criteria to assess bowel habits but asked about defecation frequency alone. Accordingly, patients with functional constipation may be classified into a non-constipated group. Fourth, colon length was measured by one author (KU). This could result in a lack of objective analysis because colon length was manually estimated in case of failure of the automatic process.

In conclusion, this study has clearly demonstrated that bowel habits (defecation frequency) and gender both correlate with the length of the entire colon measured by CT colonography, and in particular, the proximal colon. Proximal colon length might be a new surrogate biomarker to objectively diagnose slow transit constipation.

Supplementary Information The online version contains supplementary material available at https://doi.org/10.1007/s11604-021-01204-7.

Acknowledgements The Statistical Consulting Service at Health Data Science Research Section, Tokyo Metropolitan Institute of Gerontology, and Clinical Research Center at Fukushima Medical University, in particular Dr. Noriko Tanaka, helped with the interpretation of the results from statistical analysis in this study. The authors would like to

Table 5 Reports measuring the entire colon length using CT colonography

\begin{tabular}{|c|c|c|c|c|c|c|c|c|c|}
\hline First author & $\begin{array}{l}\text { Publication } \\
\text { year }\end{array}$ & Software & $N$ & Indication & Nationality & Female/male & $\begin{array}{l}\text { Age, year } \\
(\text { mean } \pm S D)\end{array}$ & $\begin{array}{l}\text { Colon } \\
\text { length, } \mathrm{cm} \\
(\text { mean } \pm \mathrm{SD})\end{array}$ & Range \\
\hline \multirow[t]{2}{*}{$\begin{array}{l}\text { Hanson et al. } \\
\text { [17] }\end{array}$} & \multirow[t]{2}{*}{2007} & \multirow[t]{2}{*}{ Viatronix } & 100 & Incomplete CS & American & $59 / 41$ & $63.4 \pm 10.6$ & $210.8 \pm 38.2$ & \multirow[t]{2}{*}{$\begin{array}{l}\text { Maxi- } \\
\quad \text { mum }>3 \mathrm{~m}\end{array}$} \\
\hline & & & 100 & Complete CS & American & $40 / 60$ & $58.2 \pm 7.9$ & $167.0 \pm 20.8$ & \\
\hline $\begin{array}{l}\text { Duncan et al. } \\
\text { [18] }\end{array}$ & 2009 & Viatronix & 338 & Screening & American & $122 / 216$ & $58 \pm 7.0$ & 189 & 75 to $257 \mathrm{~cm}$ \\
\hline $\begin{array}{l}\text { Khashab et al. } \\
\text { [21] }\end{array}$ & 2009 & Viatronix & 505 & Screening & American & $266 / 239$ & $56.6 \pm 7.3$ & $189.5 \pm 26.3$ & 120 to $299 \mathrm{~cm}$ \\
\hline $\begin{array}{l}\text { Eickhoff et al. } \\
\text { [19] }\end{array}$ & 2010 & Viatronix & 100 & Screening & American & $40 / 60$ & $58.2 \pm 7.9$ & $167.0 \pm 20.8$ & $\mathrm{n} / \mathrm{a}$ \\
\hline \multirow{2}{*}{$\begin{array}{l}\text { Nagata et al. } \\
\text { [31] }\end{array}$} & \multirow[t]{2}{*}{2013} & \multirow[t]{2}{*}{ AZE } & 650 & \multirow[t]{2}{*}{ Screening } & American & $335 / 315$ & 58.9 & $158.2 \pm 21.7$ & $\mathrm{n} / \mathrm{a}$ \\
\hline & & & 650 & & Japanese & $321 / 329$ & 59.6 & $154.7 \pm 20.4$ & $\mathrm{n} / \mathrm{a}$ \\
\hline Present study & 2020 & AZE & 295 & FIT positive & Japanese & $141 / 154$ & $58 \pm 11.0$ & $150.3 \pm 18.5$ & $\begin{array}{l}109.5 \text { to } \\
195.9 \mathrm{~cm}\end{array}$ \\
\hline
\end{tabular}

Report by Punwani et al. [20] is not cited because data on entire colon length in all patients are not available

$S D$ standard deviation, $C S$ colonoscopy, FIT fecal immunochemical test, $n / a$ not available 
thank Rika Ishizuka of Aizu Medical Center for providing administrative supports.

Funding None.

\section{Declarations}

Conflict of interest The authors declare that they have no conflict of interest.

Ethical approval All procedures performed in this study were in accordance with the ethical standards of the institutional and with the Helsinki Declaration. Written informed consent was obtained from all patients. The study protocol was approved by the Institutional Review Board of all seven participating institutions (Jichi Medical University, Kameda Medical Center Makuhari, Nagasaki Kamigoto Hospital, Hokkaido Gastroenterology Hospital, Matsuda Hospital, Kameda Medical Center and Otaru Kyokai Hospital).

Open Access This article is licensed under a Creative Commons Attribution 4.0 International License, which permits use, sharing, adaptation, distribution and reproduction in any medium or format, as long as you give appropriate credit to the original author(s) and the source, provide a link to the Creative Commons licence, and indicate if changes were made. The images or other third party material in this article are included in the article's Creative Commons licence, unless indicated otherwise in a credit line to the material. If material is not included in the article's Creative Commons licence and your intended use is not permitted by statutory regulation or exceeds the permitted use, you will need to obtain permission directly from the copyright holder. To view a copy of this licence, visit http://creativecommons.org/licenses/by/4.0/.

\section{References}

1. Peppas G, Alexiou VG, Mourtzoukou E, Falagas ME. Epidemiology of constipation in Europe and Oceania: a systematic review. BMC Gastroenterol. 2008;8:5.

2. Suares NC, Ford AC. Prevalence of, and risk factors for, chronic idiopathic constipation in the community: systematic review and meta-analysis. Am J Gastroenterol. 2011;106:1582-91 (quiz 1581, 1592)

3. Higgins PD, Johanson JF. Epidemiology of constipation in North America: a systematic review. Am J Gastroenterol. 2004;99:750-9.

4. Wald A, Scarpignato C, Kamm MA, Mueller-Lissner S, Helfrich I, Schuijt C, et al. The burden of constipation on quality of life: results of a multinational survey. Aliment Pharmacol Ther. 2007;26:227-36.

5. Bernstein C, Thorn M, Monsees K, Spell R, O'Connor JB. A prospective study of factors that determine cecal intubation time at colonoscopy. Gastrointest Endosc. 2005;61:72-5.

6. Anderson JC, Messina CR, Cohn W, Gottfried E, Ingber S, Bernstein $\mathrm{G}$, et al. Factors predictive of difficult colonoscopy. Gastrointest Endosc. 2001;54:558-62.

7. Rex DK, Chen SC, Overhiser AJ. Colonoscopy technique in consecutive patients referred for prior incomplete colonoscopy. Clin Gastroenterol Hepatol. 2007;5:879-83.

8. Bryant J. Observations upon the growth and length of the human intestine. Am J Med. 1924;167:499-519.

9. Phillips M, Patel A, Meredith P, Will O, Brassett C. Segmental colonic length and mobility. Ann R Coll Surg Engl. 2015;97:439-44.
10. Raahave D. Dolichocolon revisited: an inborn anatomic variant with redundancies causing constipation and volvulus. World $\mathrm{J}$ Gastrointest Surg. 2018;10:6-12.

11. Saunders BP, Phillips RK, Williams CB. Intraoperative measurement of colonic anatomy and attachments with relevance to colonoscopy. Br J Surg. 1995;82:1491-3.

12. Raahave D, Christensen E, Loud FB, Knudsen LL. Correlation of bowel symptoms with colonic transit, length, and faecal load in functional faecal retention. Dan Med Bull. 2009;56:83-8.

13. Saunders BP, Halligan S, Jobling C, Fukumoto M, Moussa ME, Williams CB, et al. Can barium enema indicate when colonoscopy will be difficult? Clin Radiol. 1995;50:318-21.

14. Madiba TE, Haffajee MR, Sikhosana MH. Radiological anatomy of the sigmoid colon. Surg Radiol Anat. 2008;30:409-15.

15. Sadahiro S, Ohmura T, Yamada Y, Saito T, Taki Y. Analysis of length and surface area of each segment of the large intestine according to age, sex and physique. Surg Radiol Anat. 1992;14:251-7.

16. Waldron D, Bowes KL, Kingma YJ, Cote KR. Colonic and anorectal motility in young women with severe idiopathic constipation. Gastroenterology. 1988;95:1388-94.

17. Hanson ME, Pickhardt PJ, Kim DH, Pfau PR. Anatomic factors predictive of incomplete colonoscopy based on findings at CT colonography. AJR Am J Roentgenol. 2007;189:774-9.

18. Duncan JE, McNally MP, Sweeney WB, Gentry AB, Barlow DS, Jensen DW, et al. CT colonography predictably overestimates colonic length and distance to polyps compared with optical colonoscopy. AJR Am J Roentgenol. 2009;193:1291-5.

19. Eickhoff A, Pickhardt PJ, Hartmann D, Riemann JF. Colon anatomy based on CT colonography and fluoroscopy: impact on looping, straightening and ancillary manoeuvres in colonoscopy. Dig Liver Dis. 2010;42:291-6.

20. Punwani S, Halligan S, Tolan D, Taylor SA, Hawkes D. Quantitative assessment of colonic movement between prone and supine patient positions during CT colonography. Br J Radiol. 2009;82:475-81.

21. Khashab MA, Pickhardt PJ, Kim DH, Rex DK. Colorectal anatomy in adults at computed tomography colonography: normal distribution and the effect of age, sex, and body mass index. Endoscopy. 2009;41:674-8.

22. Ohgo H, Imaeda H, Yamaoka M, Yoneno K, Hosoe N, Mizukami T, et al. Irritable bowel syndrome evaluation using computed tomography colonography. World J Gastroenterol. 2016;22:9394-9.

23. Utano K, Nagata K, Honda T, Mitsushima T, Yasuda T, Kato T, et al. Diagnostic performance and patient acceptance of reducedlaxative CT colonography for the detection of polypoid and nonpolypoid neoplasms: a multicenter prospective trial. Radiology. 2017;282:399-407.

24. Nagata K, Fujiwara M, Shimamoto T, Iida N, Mogi T, Mitsushima $\mathrm{T}$. Colonic distention at CT colonography: randomized evaluation of both IV hyoscine butylbromide and automated carbon dioxide insufflation. AJR Am J Roentgenol. 2015;204:76-82.

25. Heredia DJ, Dickson EJ, Bayguinov PO, Hennig GW, Smith TK. Colonic elongation inhibits pellet propulsion and migrating motor complexes in the murine large bowel. J Physiol. 2010;588:2919-34.

26. Heredia DJ, Grainger N, McCann CJ, Smith TK. Insights from a novel model of slow-transit constipation generated by partial outlet obstruction in the murine large intestine. Am J Physiol Gastrointest Liver Physiol. 2012;303:G1004-1016.

27. Yik YI, Cook DJ, Veysey DM, Tudball CF, Cain TM, Southwell $\mathrm{BR}$, et al. How common is colonic elongation in children with slow-transit constipation or anorectal retention? J Pediatr Surg. 2012;47:1414-20. 
28. Lacy BE, Mearin F, Chang L, Chey WD, Lembo AJ, Simren M, et al. Bowel Disorders. Gastroenterology. 2016;150:1393-407.

29. Lewis SJ, Heaton KW. Stool form scale as a useful guide to intestinal transit time. Scand J Gastroenterol. 1997;32:920-4.

30. Muller-Lissner SA, Kamm MA, Scarpignato C, Wald A. Myths and misconceptions about chronic constipation. Am J Gastroenterol. 2005; 100:232-42.

31. Nagata K, Tajiri H, Mitsushima T, Utano K, Takabayashi K, Watanabe T, et al. Colorectal length in Japanese and American asymptomatic adults based on CT colonography. Gastroenterol Endosc. 2013;55:435-44 (Japanese with English abstract).

Publisher's Note Springer Nature remains neutral with regard to jurisdictional claims in published maps and institutional affiliations. 